\title{
Illumination and Resolution Analyses on Marine Seismic Data Acquisitions by the Adjoint Wavefield Method
}

\author{
Kun-Sung $\mathrm{Li}^{1}$ and How-Wei Chen ${ }^{2, *}$ \\ ${ }^{1}$ Institute of Seismology, National Chung-Cheng University, Chiayi, Taiwan, ROC \\ ${ }^{2}$ Institute of Geophysics, National Central University, Jhongli, Taiwan, ROC
}

Received 10 May 2012, accepted 15 June 2012

\begin{abstract}
We applied a wave-equation based adjoint wavefield method for seismic illumination and resolution analyses. A twoway wave-equation is used to calculate directional and diffracted energy fluxes for waves propagating between sources and receivers to the subsurface target. The first-order staggered-grid pressure-velocity formulation, which lacks the characteristic of being self-adjoint is further validated and corrected to render the modeling operator before its practical application. Despite most published papers on synthetic kernel research, realistic applications to two field experiments are demonstrated and emphasize its practical needs. The Fréchet sensitivity kernels are used to quantify the target illumination conditions. For realistic illumination measurements and resolution analyses, two completely different survey geometries and nontrivial pre-conditioning strategies based on seismic data type are demonstrated and compared. From illumination studies, particle velocity responses are more sensitive to lateral velocity variations than pressure records. For waveform inversion, the more accurately estimated velocity model obtained the deeper the depth of investigation would be reached. To achieve better resolution and illumination, closely spaced OBS receiver interval is preferred. Full waveform approach potentially provides better depth resolution than ray approach. The quantitative analyses, a by-product of full waveform inversion, are useful for quantifying seismic processing and depth migration strategies.
\end{abstract}

Key words: Illumination analysis, Resolution analysis, Sensitivity kernels, Pressure-velocity wave equation, Reciprocity, Adjoint method, Self-adjoint operator

Citation: Li, K. S. and H. W. Chen, 2012: Illumination and resolution analyses on marine seismic data acquisitions by the adjoint wavefield method. Terr. Atmos. Ocean. Sci., 23, 621-632, doi: 10.3319/TAO.2012.06.15.01(T)

\section{INTRODUCTION}

Waveform modeling and inversion are important, active and up-front research topics that utilize different types of wave responses from a complex 2D and 3D geological media. However, if spatiotemporal properties of the collected seismic data are aliased, usually more severe in space than in the time domain, the migrated depth section or inverted velocity structure will have very limited or even distorted image. The imaged subsurface target could be affected by many factors including limited acquisition geometry, available source/receiver instrument types, driving frequency band, difference in shooting patterns, complex overburden structure and the dip angle of the reflector. Therefore, angledependent illumination, resolution information and the as-

\footnotetext{
* Corresponding author

E-mail:hwchen@earth.ncu.edu.tw
}

sociated correction strategy may be needed.

Traditional illumination and resolution analyses can be implemented by the ray-based method (Chen 1998; Schneider and Winbow 1999; Bear et al. 2000); common reflection (or conversion) point (CRP or CCP, Zhu 2000, 2002) or Fresnel zone (Muerdter and Ratcliff 2001a, b; Muerdter et al. 2001). Standard ray (Červený and Klimeš 1984), geometric ray (Chen 1998) or dynamic ray tracing (Červený and Pšenčík 1983) can be used to calculate energy propagation along the source-target-receiver path. These approaches are all ray-based illumination analysis which relies on a smoothly varying velocity model, distribution of ray paths, potential coverage on the target horizons, offset, hit counts and are often demonstrated with checkerboard tests.

Although the ray-based analysis can handle irregular acquisition geometry and laterally varying velocity models, 
the high-frequency asymptotic approximation, ray path and caustic inherent in ray theory may severely limit its accuracy and sensitivity in structurally complex regions. While ray-based method is relatively efficient for target-oriented analysis, it is not a "physics-based" approach. Moreover, frequency information is not used as travel-time is the main parameter considered. Even though an ad hoc approach, the so-called fat-ray approximation (Husen et al. 2001), was introduced in order to incorporate frequency information. Once travel-time is picked, the frequency information is lost in the analysis. By exploiting a pulsed signal traveling from source to a distance receiver through seismically defined media properties, measuring waveform changes affected by multitude of different propagation paths becomes important. Therefore physical concept of wave paths, introduced by Luo and Schuster (1991) and Woodward (1992) would closely relate to Fresnel optics (Born and Wolf 1980).

Many attempts have been made through synthetic studies by applying the wave-equation based method for seismic analyses. Using common focusing point (CFP), instead of CRP or CCP analysis, Rietveld et al. (1992), Berkhout et al. (2001) and Volker et al. (2001) investigated the effect of acquisition geometry on the target illumination through migration analysis. Hoffmann (2001) used the illumination information for resolution analysis. Gelius et al. (2002) and Lecomte et al. (2003) defined a resolution function and discussed the effect from complex velocity model. Schuster and $\mathrm{Hu}$ (2000) derived an analytical solution for target point-scatter responses by assuming a homogeneous velocity model with continuous distributed sources and receivers. These results are usually restricted to simple geometries or do not provide directional information that is crucial for target oriented imaging. Rickett (2003) developed a normalization scheme to compensate for the effect of irregular illumination. Based on a one-way wave-equation, Xie et al. (2006) utilized generalized screen propagator and local plane-wave analysis methods to properly cope multiplescattering phenomena, including focusing/defocusing, diffraction and wave-interference effects but ignored reverberations between heterogeneous layers.

In this paper, two practical contributions are made based on kernel studies through the adjoint wavefield method. We validate and correct the non-self-adjoint property involved in the first-order pressure-velocity formulation for numerical modeling. The propagator based on two-way wave equations is implemented to extrapolate the wavefields forward from sources and backward propagate the residual wavefields from receivers to the target region. Based on kernel construction for illumination measurements and resolution analyses, the concepts of Fresnel optics are used in realizing main energy fluxes and diffraction pattern caused by the local velocity perturbations. Taking the advantage of not being limited to the smoothly varying velocity model, the proposed illumination analysis is applied and compared their resolution limits for both $2 \mathrm{D}$ and $3 \mathrm{D}$ marine seismic data acquisition surveys conducted in 2008 and 2009 respectively.

\section{ILLUMINATION ANALYSIS WITH ADJOINT STATE METHOD}

Following Xie et al. (2006), the wave equation based algorithm for illumination analysis is briefly outlined. Using the multiple forward-scattering followed by single-backward scattering approximation, the full information content in the recorded seismogram can be represented through Green's function by $3 \mathrm{D}$ volume integral over sources and receivers.

$P\left(\mathbf{r}, \mathbf{r}_{\mathrm{s}}, \mathbf{r}_{\mathbf{R}}\right)=2 k_{o}^{2} \int_{V} m\left(\mathbf{r}^{\prime}\right) G\left(\mathbf{r}, \mathbf{r}^{\prime} ; \mathbf{r}_{\mathrm{s}}\right) G\left(\mathbf{r}, \mathbf{r}^{\prime} ; \mathbf{r}_{\mathbf{R}}\right) d V\left(\mathbf{r}^{\prime}\right)$

where $\mathbf{r}$ is spatial position vector within $V(\mathbf{r}), P(\mathbf{r})$ is the computed quantity from superposition of seismic wave responses of Green's function $G\left(\mathbf{r}, \mathbf{r}^{\prime} ; \mathbf{r}_{\mathrm{s}}\right)$ excited by an arbitrary point source $\mathbf{r}_{\mathrm{S}}$ over $3 \mathrm{D}$ computational domain. The Fréchet derivative is $m\left(\mathbf{r}^{\prime}\right)=\delta c\left(\mathbf{r}^{\prime}\right) / c\left(\mathbf{r}^{\prime}\right)$ where $\delta c\left(\mathbf{r}^{\prime}\right)$ is the velocity perturbation, $c\left(\mathbf{r}^{\prime}\right)$ is the velocity, $\boldsymbol{k}_{0}=\omega / c_{0}(\mathbf{r})$ is the background wavenumber, $c_{0}(\mathbf{r})$ is the local background reference velocity and $\omega$ is angular frequency. We use a two-way pressure-velocity wave-equation based propagator to calculate Green's functions.

To calculate illumination, a fictitious plane reflector with a dipping angle and a unit (or constant) reflectivity is usually required (Muerdter and Ratcliff 2001a). Using plane-wave superposition principle, we can generalize the idea into an arbitrary non-flat reflector. The target illumination response function [Xie et al. 2006: Fig. 1 and Eq. (5); Virieux and Operto 2009: Fig. 4] is defined as

$D\left(\mathbf{r}, \mathbf{r}_{\mathrm{s}}, \mathbf{r}_{\mathrm{R}}\right)=\iint A\left(\mathbf{r}, \mathbf{K}_{\mathrm{s}}, \mathbf{K}_{\mathrm{R}} ; \mathbf{r}_{\mathrm{s}}, \mathbf{r}_{\mathrm{R}}\right) M\left(\mathbf{r}, \mathbf{k}_{\mathrm{s}}+\mathbf{k}_{\mathrm{R}}\right) d \mathbf{K}_{\mathrm{R}} d \mathbf{K}_{\mathrm{s}}$

where $M(\mathbf{r}, \mathbf{k})=|m(\mathbf{r}, \mathbf{k})|$, and

$A\left(\mathbf{r}, \mathbf{K}_{\mathrm{s}}, \mathbf{K}_{\mathrm{R}} ; \mathbf{r}_{\mathrm{s}}, \mathbf{r}_{\mathrm{R}}\right)=2 k_{0}^{2} I\left(\mathbf{K}_{\mathrm{s}}, \mathbf{r} ; \mathbf{r}_{\mathrm{s}}\right) I\left(\mathbf{K}_{\mathrm{R}}, \mathbf{r} ; \mathbf{r}_{\mathrm{R}}\right)$

is the local illumination matrix of the source-receiver pair $\left(\mathbf{r}_{\mathrm{S}}, \mathbf{r}_{\mathrm{R}}\right)$. The equations

$I\left(\mathbf{K}_{\mathrm{s}}, \mathbf{r} ; \mathbf{r}_{\mathrm{s}}\right)=G\left(\mathbf{K}_{\mathrm{s}}, \mathbf{r} ; \mathbf{r}_{\mathrm{s}}\right) G^{*}\left(\mathbf{K}_{\mathrm{s}}, \mathbf{r} ; \mathbf{r}_{\mathrm{s}}\right)$

and

$I\left(\mathbf{K}_{\mathrm{R}}, \mathbf{r} ; \mathbf{r}_{\mathrm{R}}\right)=G\left(\mathbf{K}_{\mathrm{R}}, \mathbf{r} ; \mathbf{r}_{\mathrm{R}}\right) G^{*}\left(\mathbf{K}_{\mathrm{R}}, \mathbf{r} ; \mathbf{r}_{\mathrm{R}}\right)$

are the mean squares of Green's functions, which are proportional to energy fluxes form the source and receiver to the target, respectively. The $*$ denotes complex conjugate, 
$\mathbf{K}$ is the horizontal wavenumber with $\mathbf{k}=\mathbf{K}+\mathbf{k}_{\mathbf{z}} \hat{\mathbf{e}}_{\mathbf{z}}$. Given the local reference $c_{0}(\mathbf{r})$, the $\mathbf{k}_{\mathbf{z}}$ can be determined from its horizontal component via $\boldsymbol{k}_{z}=\sqrt{\boldsymbol{k}_{o}^{2}-\boldsymbol{K}^{2}}$.

For a system composed of multiple sources and receivers, the illumination responses can be calculated by stacking all contributions from individual source-receiver pair.

$$
\begin{aligned}
& D(\mathbf{r})=\sum_{\mathbf{r}_{\mathrm{S}}} \sum_{\mathbf{r}_{\mathrm{R}}} D\left(\mathbf{r}, \mathbf{r}_{\mathrm{s}}, \mathbf{r}_{\mathrm{R}}\right) \\
& =\iint \sum_{\mathbf{r}_{\mathrm{s}}} \sum_{\mathbf{r}_{\mathrm{R}}} A\left(\mathbf{r}, \mathbf{K}_{\mathrm{s}}, \mathbf{K}_{\mathrm{R}} ; \mathbf{r}_{\mathrm{s}}, \mathbf{r}_{\mathbf{R}}\right) M\left(\mathbf{r}, \mathbf{k}_{\mathrm{s}}+\mathbf{k}_{\mathbf{R}}\right) d \mathbf{K}_{\mathbf{R}} d \mathbf{K}_{\mathrm{s}}
\end{aligned}
$$

For a given acquisition geometry and background velocity, the matrix $A\left(\mathbf{r}, \mathbf{K}_{\mathbf{s}}, \mathbf{K}_{\mathbf{R}}\right)$ is composed of all possible local scattering events that contribute to target illumination. The integral sums the energy that can actually contribute to a particular target and gives the illumination response at location $\mathbf{r}$. Thus, all effects involved in the acquisition configuration, the background velocity and perturbation closely associated with surface topography, layer boundary, target geometry and reflectivity strength are all included in the calculation. The total illumination response function, $D_{T}(\mathbf{r})$, is calculated by sums of energy from all possible scattering contributions for all source-receiver pairs.

Similar theory used in ocean acoustic studies, following Huygens-Fresnel principle and invoking reciprocity, the acoustic diffraction pattern $D_{\text {pattern }}$ or equivalently 'the effective pressure (Fréchet derivative) sensitivity kernel' can be computed as the product of both Green's functions at the receiver $\mathbf{r}_{\mathbf{R}}$ and at the source $\mathbf{r}_{\mathrm{S}}$ (Iturbe et al. 2009; Virieux and Operto 2009).

$$
D_{\text {Pattern }}\left(\mathbf{r}, \mathbf{r}_{\mathrm{S}}, \mathbf{r}_{\mathrm{R}}\right)=G\left(\mathbf{r}, \mathbf{r}_{\mathrm{S}}\right) G\left(\mathbf{r}, \mathbf{r}_{\mathrm{R}}\right)
$$

The computation of diffraction pattern can be explicitly constructed through the adjoint-state wavefield (AW) operator. The procedure involves reverse-time propagation of recorded data and cross-correlating with the corresponding forward propagating wave fields from source (McMechan 1982; Claerbout 1985).

\section{VALIDATION ON SELF-ADJOINT OPERATOR FOR MULTI-COMPONENTS OBS DATA}

Using the pressure-velocity formulation based wave propagation engine to construct its associated effective sensitivity kernels may vary depending on the type of data used. For simulating observed vertical component data with pressure (air-gun or explosive) source synthesis, the spatial reciprocity relationship between vertical particle velocity wavefields generated by explosion and corresponding pressure wavefields generated by a vertical force have to be verified first. Chen $(1996,1999)$ and Operto et al. (2006) in- dicated that the fundamental relationship between explosive pressure sources is equivalent to two perpendicular vector dipoles with a fixed arm. Base on reciprocity principle, one can match pressure wavefields generated by a vertical force with vertical velocity wavefields generated by an explosion.

Figure 1 documents the validation procedures on utilizing the self-adjoint operator through first-order pressure-velocity wave equation formulation involved in ocean bottom seismograph (OBS) data and survey geometry. The validation on reciprocity principle and consistency is important when full waveform modeling and inversion is implemented. Separate tests (Figs. 1a to c) are conducted to validate non-self-adjoint operator and the correction made in utilizing elastodynamic wave equation. Each figure contains seismograms from forward simulation (left panels), adjoint data for backward propagation from the receiver position (center panels) and the corresponding sensitivity kernels (right panels). For marine OBS survey, air-gun explosive (E) source, usually located a few meters below sea surface, is detonated and different types of seismic responses can be recorded by OBS deployed at the sea floor. Multi-attribute seismic responses recorded by hydrophone pressure $(\mathrm{P})$ sensor, horizontal $\left(\mathrm{V}_{\mathrm{x}}\right)$ and vertical $\left(\mathrm{V}_{\mathrm{z}}\right)$ components of geophone sensors are simultaneously recorded. Through acousto- (Chen 1999), viscoacoustic (Chen 1996, 2006), viscoelastic (Kang and Chen 2003) and/or elastodynamic wave equations (Chen and McMechan 1992) approach for multi-component and multi-attribute wavefield computations, we are able to carefully check the corresponding pressure and velocity kernels from the same adjoint operator. The computed Fréchet derivative kernel based on the AW method is used to validate the characteristic of self-adjoint property through the same forward modeling engine.

To simplify the description in each figure, we formulate our test cases through $[\mathrm{X}, \mathrm{S} 2 \mathrm{R}$ (or R2S), $\mathrm{Y}]$ notation to indicate the propagation path from source $(\mathrm{S})$ to receiver $(\mathrm{R})$ or vice versa. Symbol $X$ denotes the type of excitation by explosion, horizontal force $\left(F_{x}\right)$ or vertical force $\left(F_{z}\right)$. Symbol $\mathrm{Y}$ denotes the associated type of computed seismic responses $\left(\mathrm{P}, \mathrm{V}_{\mathrm{x}}\right.$ or $\left.\mathrm{V}_{\mathrm{z}}\right)$. In addition, a schematic diagram is attached at each seismogram display in order to indicate main wave propagation direction in each testing case. Figure 1a shows that the sensitivity kernels obtained from three cases have different illumination properties including waveform amplitude, polarity and diffraction pattern. For example, Fig. 1a shows the sensitivity kernel obtained from $\mathrm{S}$ suspended at $300 \mathrm{~m}$ below sea surface and R located at the sea floor at depth of $1700 \mathrm{~m}$. The Fréchet derivative kernel shown in the top panels is computed from cross-correlation between E,S2R,P and E,R2S,P wavefields. The kernel shows the image from diffraction sums and thus indicates its diffraction pattern. For other two cases, the amount of energy, inferred from amplitude, involved in the forward simulation of particle velocity $\left(\mathrm{V}_{\mathrm{x}}, \mathrm{V}_{\mathrm{z}}\right)$ from $\mathrm{E}$ and its adjoint $\mathrm{P}$ data from 
(a)

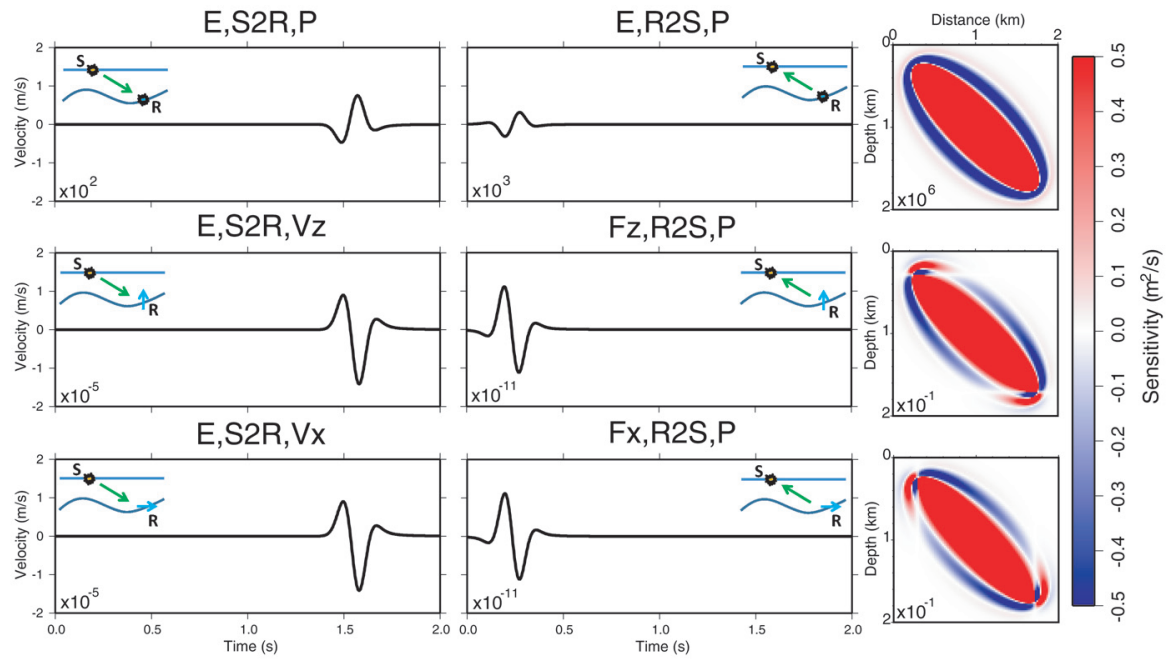

(b)
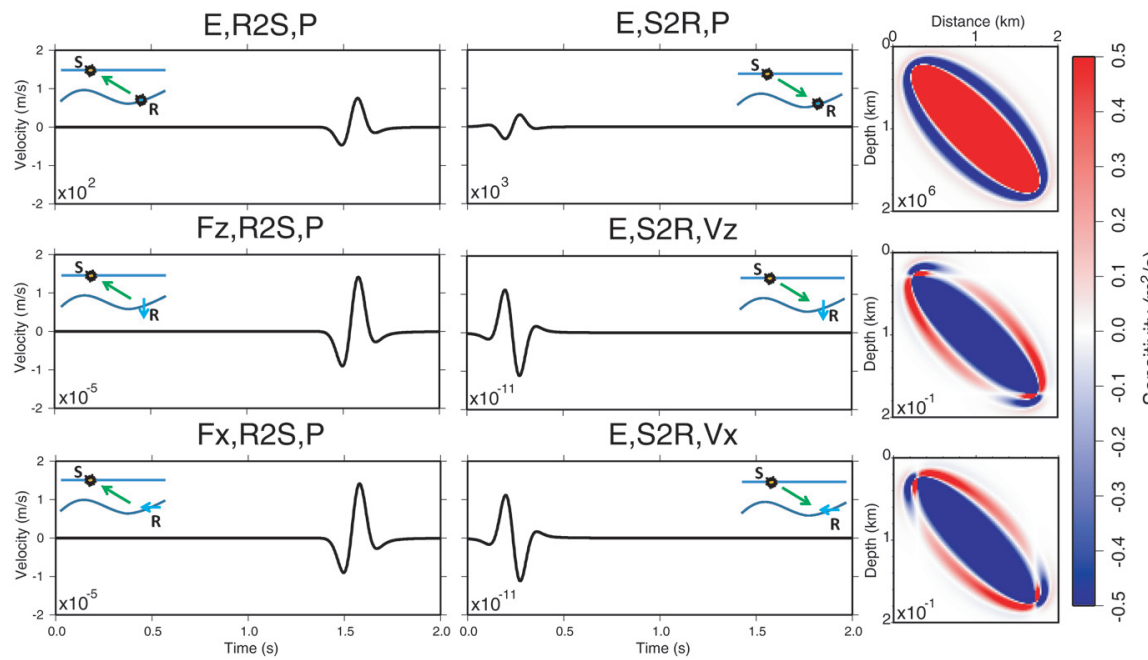

(c)
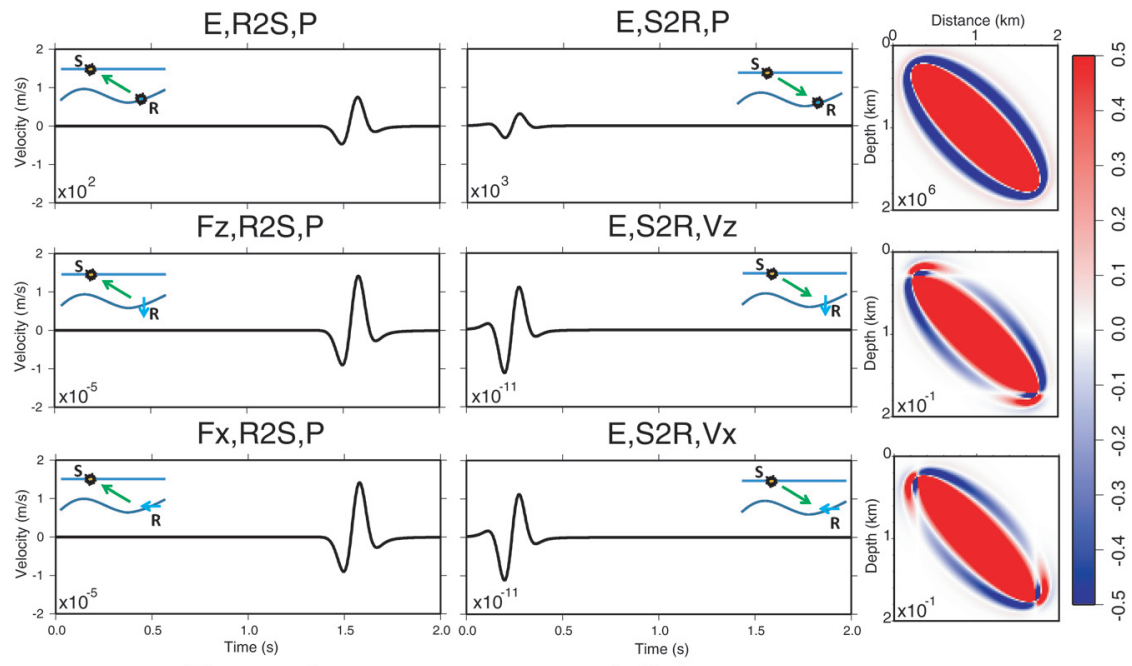

Forward

Adjoint

Fig. 1. Validation tests of the reciprocity principle based upon the pressure-velocity elastodynamic wave equation. (a) Forward propagate wavefields from source location (left panels, S2R) cross-correlated with time-reversed wavefields from receiver location (middle panels, R2S) will produce the corresponding Fréchet derivative kernels (right panels). In each test case, explosive (E) or directional dipole force $\left(\mathrm{F}_{\mathrm{x}}\right.$ or $\left.\mathrm{F}_{\mathrm{z}}\right)$ will produce pressure drop ( $\dot{\mathbf{P}})$ or two component particle velocity $\left(\mathrm{V}_{\mathrm{x}}\right.$ or $\mathrm{V}_{\mathrm{z}}$ ) seismograms. (b) The corresponding reciprocal wavefiels from receiver (left panels, $\mathrm{R} 2 \mathrm{~S}$ ) and from source (middle panels, S2R) produce reversed phase kernels (right panels) except the top panel cases for pressure responses from explosive source. (c) Corrected results. Model size is $2.0 \mathrm{~km} \times 2.0 \mathrm{~km}$ with source and OBS located at $(0.3 \mathrm{~km}, 0.3 \mathrm{~km})$ and $(1.7 \mathrm{~km}, 1.7 \mathrm{~km})$, respectively. 
dipole force $\left(F_{x}, F_{z}\right)$ are lower than the energy level shown in the top panels. Note that the non-zero value in the first Fresnel zone is mainly caused by $2 \mathrm{D}$ computation and regions close to the source and receiver locations show very distinct phase changes across iso-phase Fresnel zones.

Figure $1 \mathrm{~b}$ shows the validation results obtained from reciprocity tests. Top panels show (E,R2S,P; E,S2R,P) test case and compare the results from Fig. 1a for (E,S2R,P; $\mathrm{E}, \mathrm{R} 2 \mathrm{~S}, \mathrm{P})$. Because $\mathrm{E}$ and $\mathrm{P}$ are all isotropic and scalar quantities, thus it is not surprising to learn that the reciprocity principle is valid and the corresponding kernel appears to be the same. On the contrary, in other situations when directional force or particle velocity responses are used, the waveforms and the corresponding kernels lack the characteristic of being self-adjoint. In Fig. 1b, the kernels obtained from the middle and lower panels appear to have an opposite sign compared to the results in Fig. 1a. Our contribution, after scaling by a constant either in the residual wavefield or in the corresponding sensitivity kernel renders the modeling operator to become self-adjoint before developing the adjoint state method for illumination analysis. Figure 1c is the corresponding test cases that produced exactly the same kernels compared to the results from Fig. 1a. It is worth noting that the corresponding waveform data in both forward and adjoint operators, except top panels, exhibit reversed polarity compared to the reciprocal cases shown in Fig. 1a.

\section{APPLICATION OF MARINE SURVEYS AND DATA TYPES}

This section investigates the frequency-dependent illumination analysis related to wave paths and diffractions generated by the heterogeneous media and how sensitive the amount of velocity perturbations can be revealed by the theory discussed in the previous section. Two types of marine surveys (Fig. 2) were used to demonstrate the importance in survey design and data attributes for imaging. Multi-channel seismic (MCS) survey usually utilizes fairly narrow incidence angle for reflection arrivals mainly designed for investigating shallow crustal structural features. Figure 3 shows P-wave velocity models with two types of configurations for OBS data survey in order to compare their associated resolution limits affected by different receiver spacing and overburden structure. For a large-scale seismic survey, the strategy often requires large receiver spacing with closely spaced shot interval, mainly for recording 2D wide-angle reflection and refraction (WARR) seismic data for deep structure investigation. Such survey configuration is often used for reconnaissance survey. Another survey strategy, usually designed for 3D survey, requires more tightly spaced receiver interval. Several OBS instruments were deployed within relatively small but targeted area but still tend to collect WARR seismic data with moderate aperture for investigating shallow-to-intermediate depth structure. Such survey configuration is often used for target oriented exploration.

Figure 3 shows the comparison of two different models with scale-dependent source-receiver survey configurations for illumination and resolution analyses. The first model has an average receiver spacing of about $10 \mathrm{~km}$ while the second model has an average spacing of less than $1 \mathrm{~km}$. In addition, each model consists of two types of velocity constructions. The first type employs linearly increasing velocity gradient (LIVG) model while the second model can be derived from various velocity estimation techniques. The long-offset model has horizontal distance of $130 \mathrm{~km}$ with depth reaches $15 \mathrm{~km}$. Twelve OBSs were deployed in order to reveal largescale tectonic features. Besides the gradient model, the second velocity model is constructed from the best estimated solution from first arrival travel time (FATT; Zelt and Smith 1992) tomography. In contrast, a much smaller survey with model size of $20 \mathrm{~km} \times 7 \mathrm{~km}$ is extracted from a 3D MCS/ OBS seismic survey. The 3D survey aims to combine both MCS and OBS data for a target oriented seismic exploration of gas hydrate. For OBS survey, the target zone was further confined to within $5 \mathrm{~km}$ with eight OBSs deployed in that region. The smoothly and laterally varying velocity model was obtained from conventional semblance velocity analysis for estimating the stacking/interval velocity. The long-offset survey line (MGL0905-08) was conducted in 2009 during TAIGER (TAiwan Integrated GEodynamics Research) marine seismic survey. The intermediate-offset survey (MCS881-42) was conducted in 2008. The seismic line is part of a 3D survey conducted in the Yung-An ridge where bottom simulated reflectors (BSRs) have been identified from seismic profiles. Both surveys were conducted in offshore Southwestern Taiwan under the financial support from the Central Geological Survey, Ministry of Economic Affairs. For resolution comparison, an additional horizontal layer (marked by a red arrow) was artificially superimposed on top of each background velocity model with an additional positive velocity perturbation of $500 \mathrm{~m} \mathrm{~s}^{-1}$. The depth and thickness of the added high velocity zone (HVZ) are from 5500 to $6500 \mathrm{~m}$ for large-scale model and from 2600 to $3400 \mathrm{~m}$ for small-scale model respectively.

Figure 4 shows different illumination distribution studies for a 2D MGL0905-08 seismic section. The normalized total illumination strength which sums energy from all possible scattering combinations of all source-target-receiver responses $\left[D_{T}(\mathbf{r})\right]$ can be used for resolution analysis. Normalized amplitude varies between negative (red) and positive (blue) values indicate the different degree of illumination strength beneath the survey line. The right panels are the results based on LIVG models which are usually treated as initial models for travel-time inversion. The left panels are results based on the model derived from FATT tomography. For comparison, fine-grid models (top panels) which are consistent with streamer hydrophone spacing of 


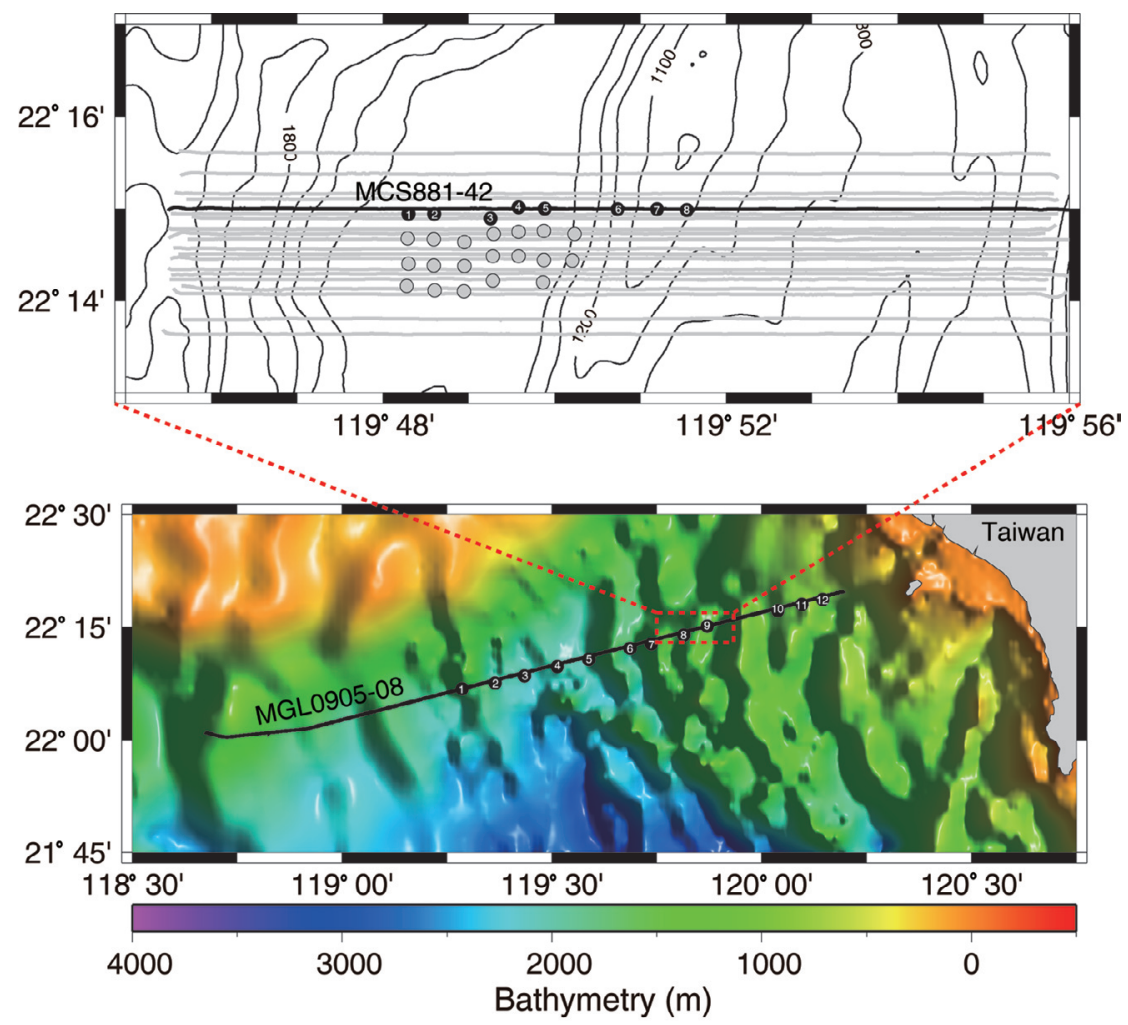

Fig. 2. Location map for long-offset survey line MGL0905-08 and an intermediate-offset survey line MCS881-42 belongs to a 3D survey. Wideangle MCS and OBS survey configuration (MGL0905-08) were designed for deep structure imaging (Deng et al. 2010). Closely spaced but more randomly distributed OBS locations are designed for a target oriented exploration survey for gas hydrate through 3D survey (Cheng et al. 2010).

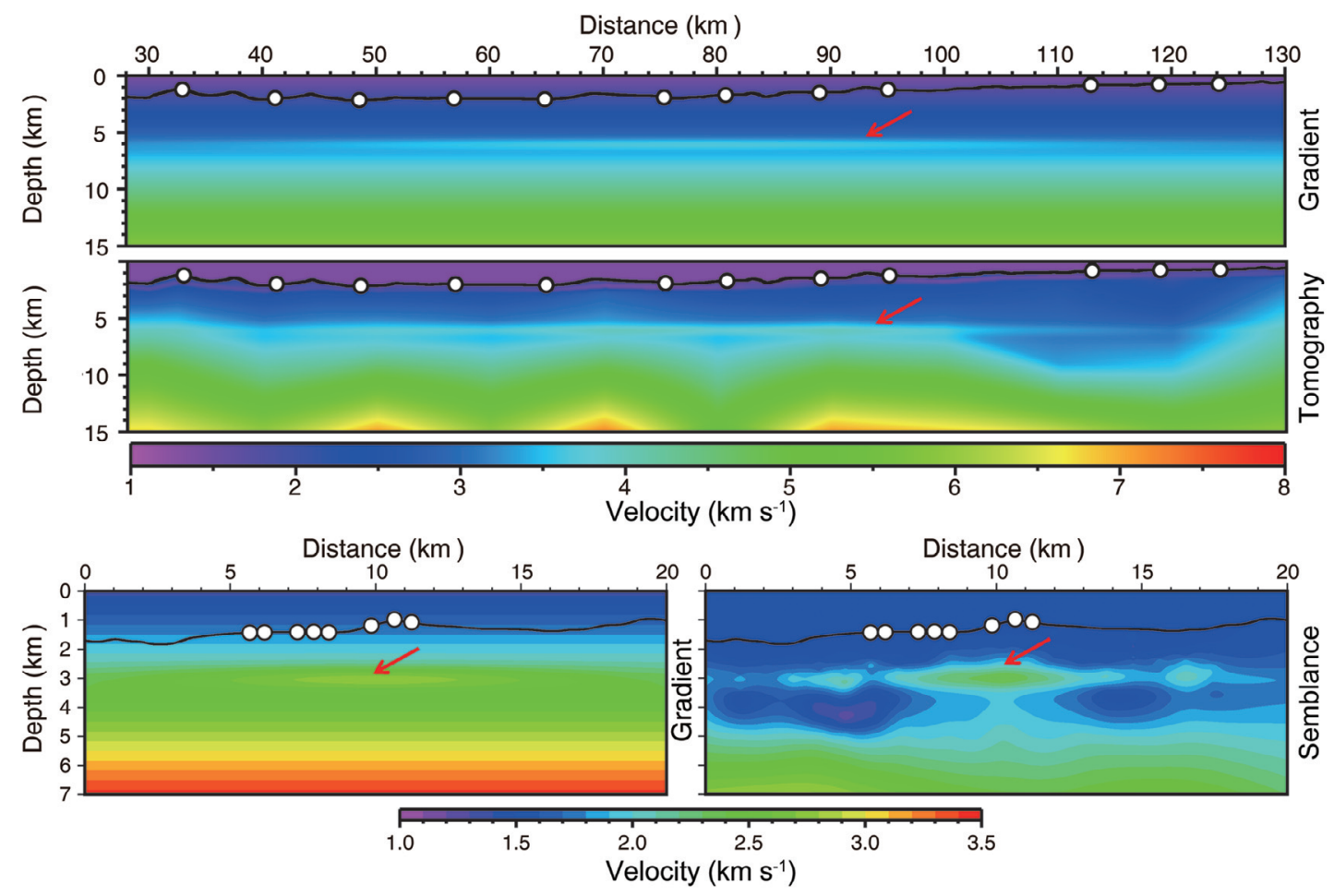

Fig. 3. P-wave velocity models for two types of survey configurations shown in Fig. 2. In each survey type, two reference models, a linearly increasing velocity gradient (LIVG) and a laterally varying velocity model, are constructed in order to compare its illumination results. The large- and intermediate-offset surveys are commonly used for marine seismic data acquisition. To compare its resolving power, each model had superimposed an additional horizontal high velocity zone (HVZ, marked by arrow). 


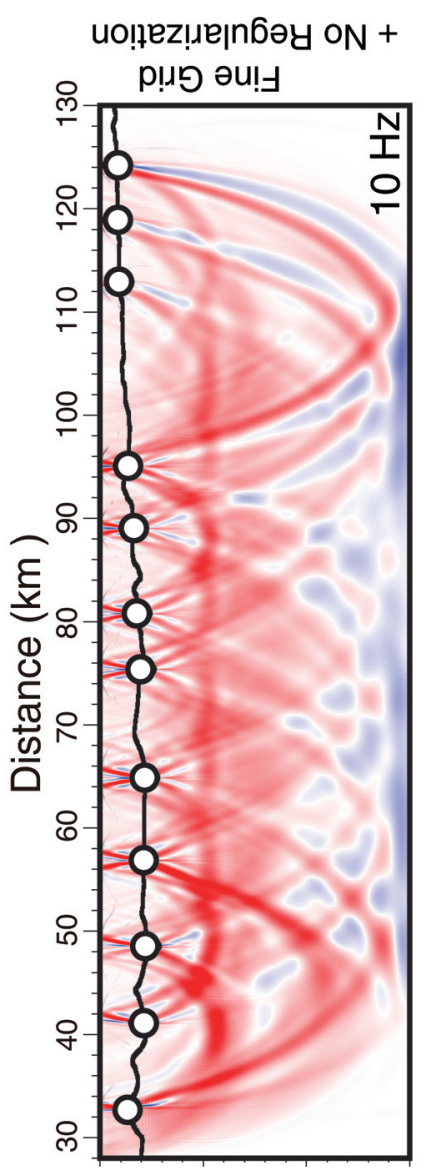

uo!̣ez!̣ejnбəy on + uo!̣ez!̣e|nбəy +
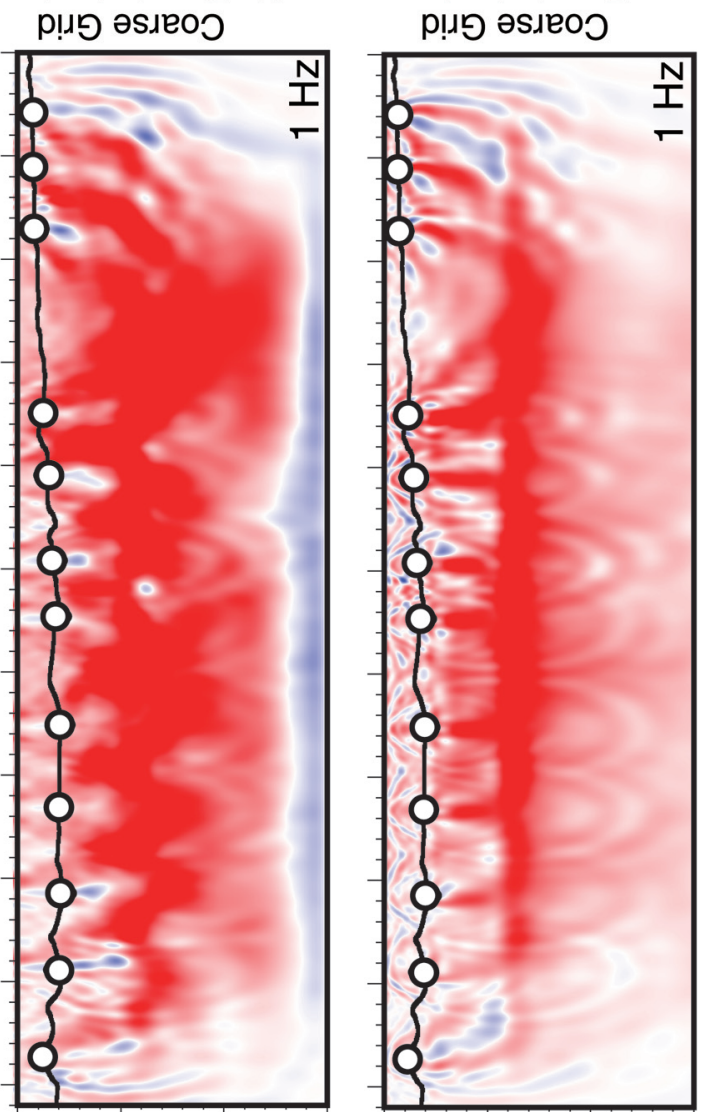

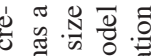
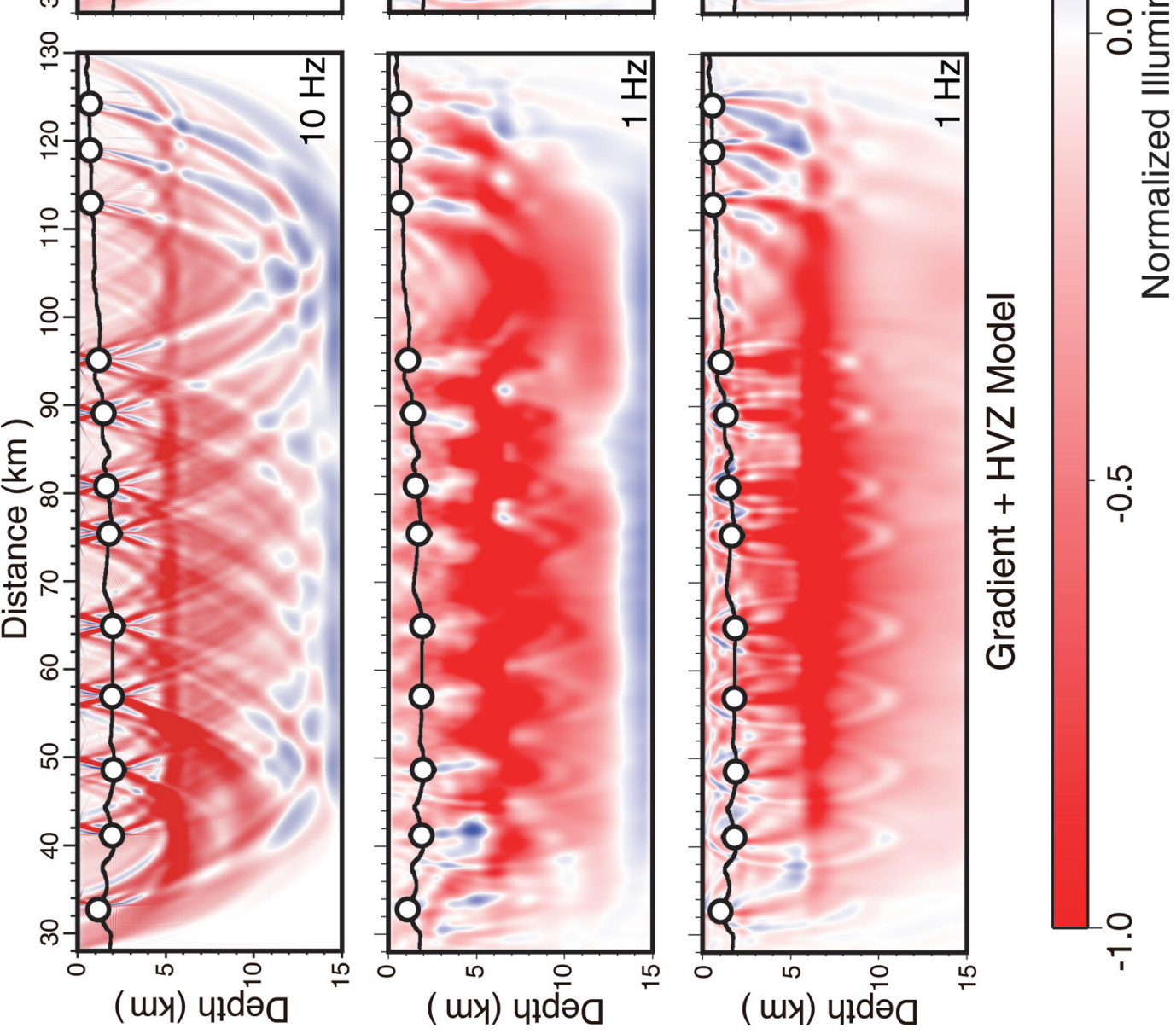

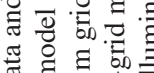

䒕缃

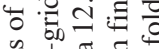

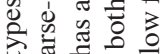

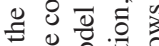

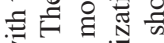

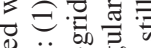

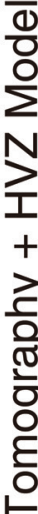

要政

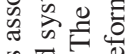

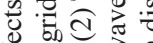

ช)

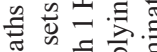

L)

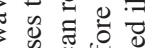

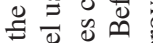

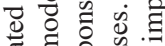

.

শิ

है

$\&$

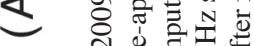

ㄷ..$\quad$ 깅의

음 离

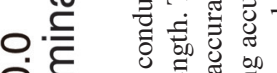

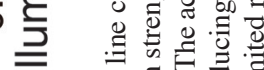

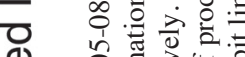

N

ত্

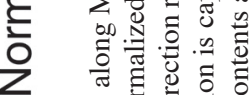

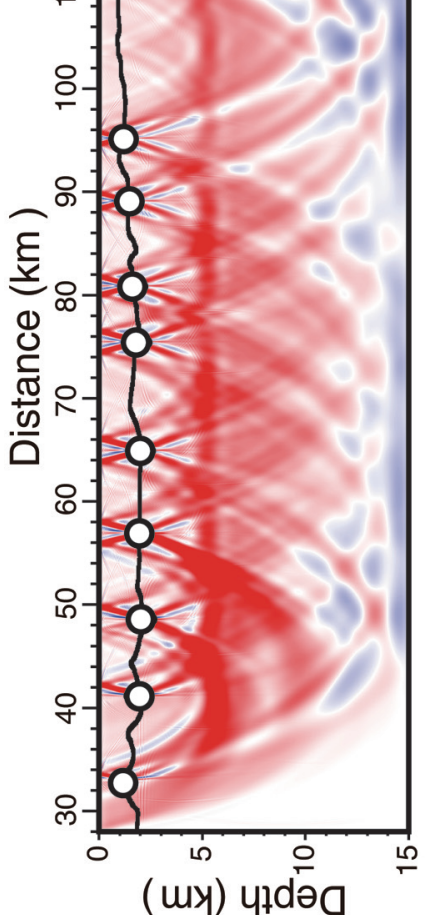

( uy) 4ұdə0

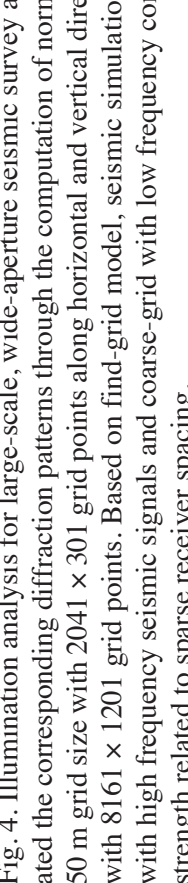


$12.5 \mathrm{~m}$ are used in order to have a high frequency content of $10 \mathrm{~Hz}$ in the computed synthetic seismograms. In contrast, to match the air gun shot interval, the coarse-grid models (middle and lower panels) have grid increment of $50 \mathrm{~m} \mathrm{ca}-$ pable of simulating $1 \mathrm{~Hz}$ responses. Illumination maps for the fine-grid model (top panels) show many low and close to zero strength regions. The zones with zero values show more apparent ray-like propagation phenomena.

Along seismic line MGL0905-08, the OBS spacing is rather sparse which varying from $\sim 5$ to $15 \mathrm{~km}$. For finegrid and high frequency contents of seismic responses, the unevenly distributed OBS locations produce undesirable variations of illumination strength and have limited resolving power in the gas hydrate bearing strata which lie several hundred meters below seafloor. Even though illumination range can be improved by using coarse-grid and low frequency seismic data (middle panels), the relative low and laterally fluctuating illumination strength obtained within a few hundred meters below seafloor still exist. Therefore, based upon pressure data unreliable analyses results could be obtained where gas hydrate is known to be abundant (Liu et al. 2006). Such situation truly reflects the potential difficulty and limitation caused by survey design strategy or owing to some practical consideration during the field deployment stage. Notice that the regions with more reliable illumination intensity (darker color in red or blue) are located between the adjacent OBS pairs and not underneath each OBS location. In addition, the "illumination holes" where low strength exists also exhibit angle-dependent illumination feature associated with each OBS location. Such spatially varying characteristic features indicate that illumination strength is function of source, receiver locations, velocity perturbation and refraction/reflection angles on the targets.

The results from quantitative illumination analysis are very useful for the follow-up seismic processing involving pre-stack depth migration for imaging and full waveform inversion for velocity refinement. For a fine-grid model, clear wave paths which correspond to seismic multiples generated by the sea floor are more apparent than those shown in the coarse-grid model. For large scale OBS survey, using coarse-grid associated with low frequency seismic content is helpful in increasing the area which could be illuminated. Thus, for this type of survey geometry with rather sparse OBS receiver spacing, data containing low frequency signature and coarse-grid model should be used for better spatial aperture coverage, but at the risk of obtaining low resolution results from all shot-target-receiver contributions. Moreover, the inserted HVZ exhibits different level of illumination intensity especially for fine-grid, high frequency data. The wider the receiver spacing, especially those two OBSs located between 95 and $115 \mathrm{~km}$, the more blurred illumination intensity would be obtained. The penetration depth also appears to be affected by the thick strata with more steeply dipping structural feature on the right hand side of the tomography model (Fig. 3). Such observed target-related and angle-dependent illumination properties will affect future survey design. However, the "checkerboard like" illumination pattern revealed from the coarsegrid model above and below the HVZ introduces additional concerns. For low frequency seismic contents, the large receiver offset will provide better depth of investigation but with lower illumination strength. Comparing results from the illumination analysis for two different velocity models, if the seismically defined media propagation speed becomes more accurate, the better and the deeper illumination can be achieved. Thus, full waveform approach can provide better resolution than ray approach.

Another important consideration is in terms of the amount of energy transmitted across the sea floor. The range-dependent reflectivity contrasts along mostly existing unconsolidated sediments, occasionally exposed by bed rock or rather thin sediments layers, provide additional uncertainty affecting our illumination analysis. Although using broadband OBS instrumentation is an ideal situation for receiving passive seismic data from teleseismic event, large receiver spacing still poses a potential problem for depth resolution. For an active source experiment, the OBS survey is designed to avoid large transmission energy loss when seismic waves traveling within the water layer. Because the air-gun source was fired a few meters below sea surface, seismic waves with rather strong amplitude traveling within the water layer will generate multiple reverberations which can mask deep reflections. The path-dependent masking effect can be partially alleviated and often the reciprocity principle is employed to ease the computational requirement in seismic modeling, migration and inversion.

Because of "checkerboard like" and irregular illumination strength pattern observed in the computed image, additional regularization (Rickett 2003; Operto et al. 2006; Guitton et al. 2010) is required. The bottom panels in Fig. 4 show the improvement after attenuating the high wavenumbers in the computed gradient by smoothing the result. The size of smoothing operator in the horizontal and vertical directions is a function of an average wavelength at a given frequency. Because normalized amplitude is used for illustration, the amount of variations before normalization is different. It is interesting to note that no matter what kind of background models (bottom panels) are used, a clear but separated elliptical shaped illumination pattern with lateral variation in its intensity becomes more apparent after regularization. Although horizontal HVZ layer becomes visible for low frequency data, more closely spaced OBS data is still preferred. Comparing illumination results between LIGV (left panels) and tomography (right panels) models, tomography model still provide better depth resolution than gradient model.

Figure 5 illustrates a completely different survey strategy with rather closely spaced OBSs for 3D target-oriented 

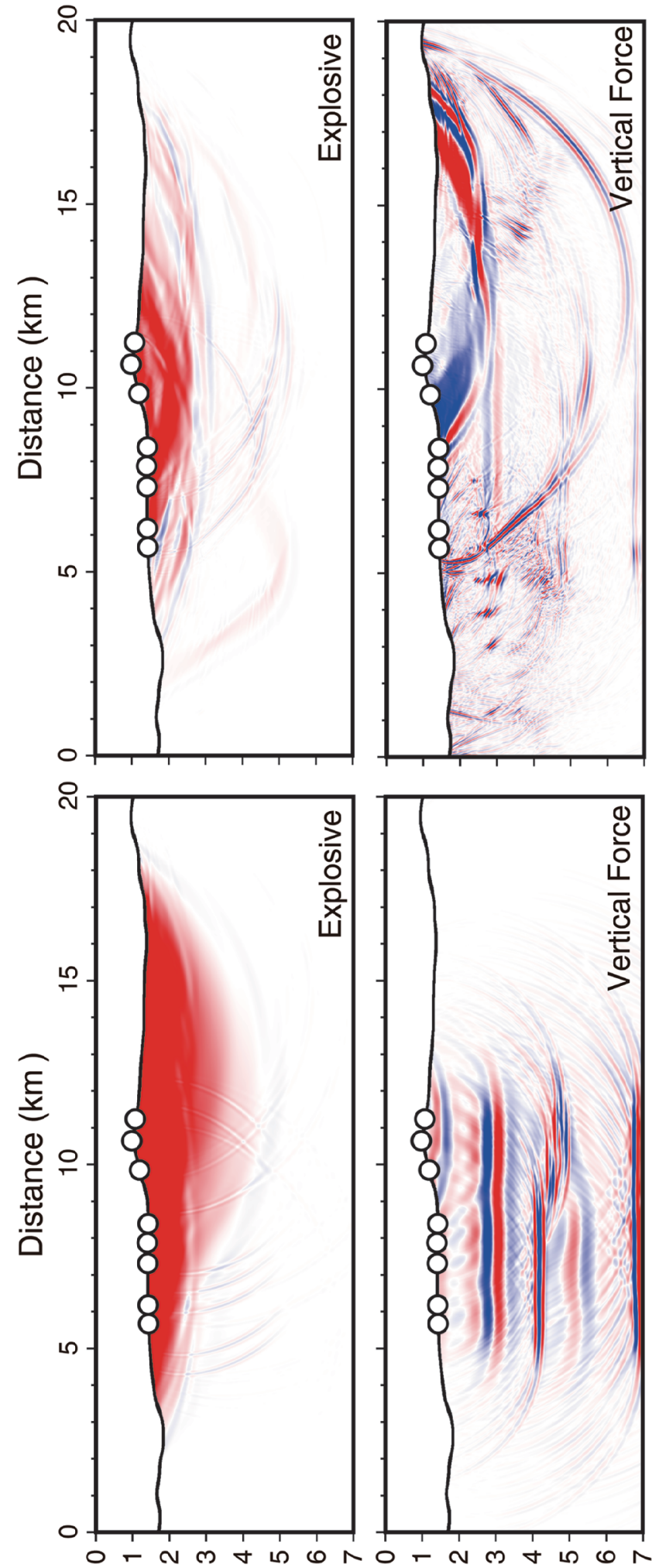

( $u y$ ) yłdəo

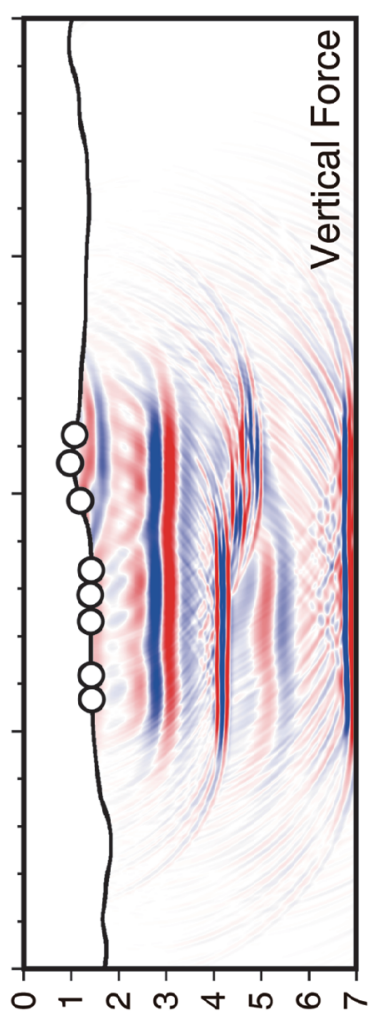

(uy) प1də0
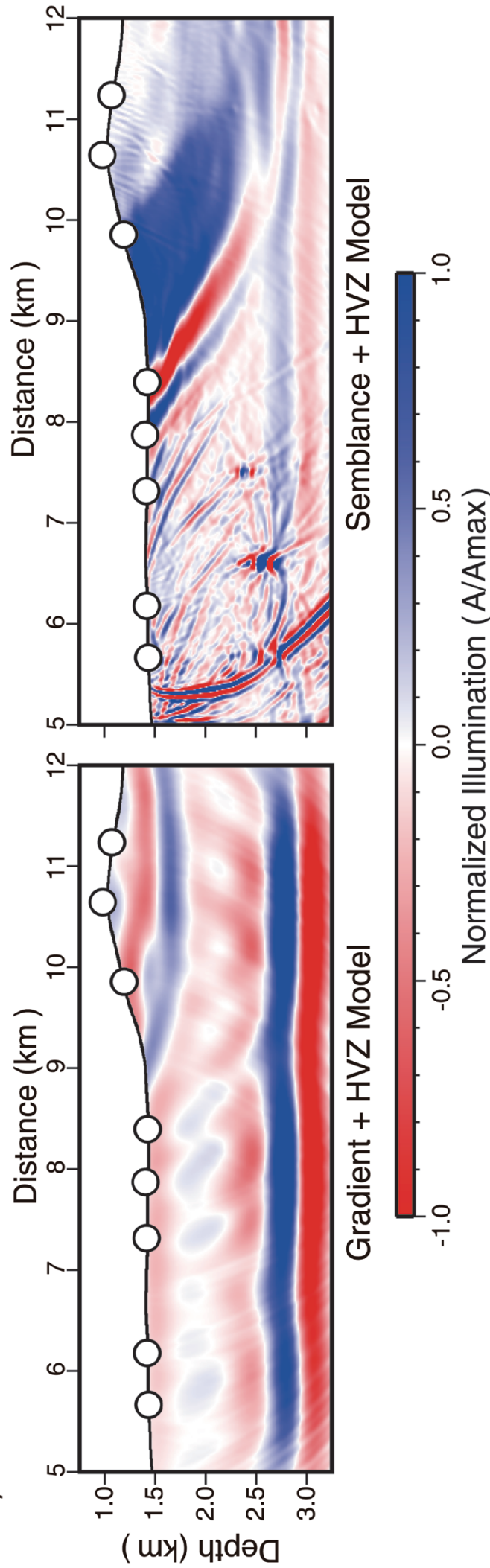

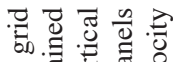

I

กิ

ส

$\Rightarrow$ ए

a

:

귱 ठำ

응 क्ष

ब1

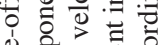

:

๑ ब 0

ए है

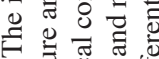

큐

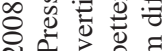

$\Xi \dot{0} \cong \dot{0}$

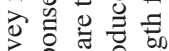

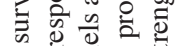

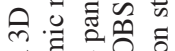

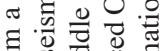

Nㅡㅁㅛ

प्र 0 क

जै $\dot{0} 0$

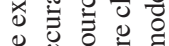

छ क्ष क्ष

ᄀ.

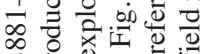

$\infty$ ○

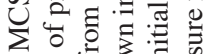

$000 . \Xi 0$

휴 क्षै

入े

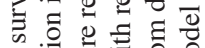

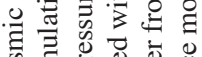

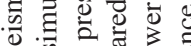

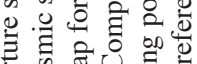

ه

을

造

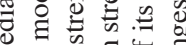

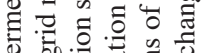

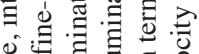

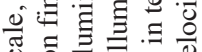

की 0.000

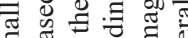

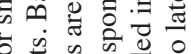

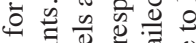

औ

त्ञ

휴올 굴

$x+9$ के

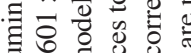

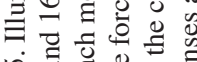

的击

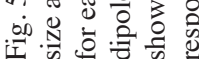


survey. In addition, we compare the illumination results and its associated resolution properties with different types of data. The top panels are the distribution of illumination strength obtained from the explosive source with pressure records for different velocity models. Lateral velocity variations estimated from semblance analysis create smaller, shallower with strong laterally varying illumination distribution while the gradient model provides more coherent illumination power. Angle-dependent illumination distribution (middle panels) is obtained from the directional vertical force with responses corresponds to the vertical component of velocity and appears to be affected by the variation of structure model. Illumination responses for the pressure field have much larger amplitude value compared to the responses created by vertical force (see amplitude level shown in Fig. 1). Moreover, the resolution power revealed from illumination analysis indicates that in spite of the source types, vertical component of velocity records appears to be more sensitive to lateral velocity changes than pressure field. The distribution of illumination strength is different in revealing the horizontal HVZ layer among different type of seismic data, source types and initial models. The lower panels of Fig. 5 show the enlarged details on the lateral changes in the computed illumination strength from pre-defined velocity distributions. Focusing, diffractions and multiples related illumination distribution are clearly visible. Thus, one would expect that using different type of data will affect the amount of computation time required for full waveform inversion.

\section{CONCLUSIONS}

The practical applications of partial derivative wavefields for constructing various sensitivity kernels from different seismic responses are demonstrated for illumination and resolution analyses. Despite various published kernel studies, mostly for synthetic case studies, our contribution clearly demonstrates its importance and practical needs before full waveform inversion can be put into practice. Seismic waveform inversion is usually an under-determined and ill-posed problem which suffers from the amount of available data, spatial aperture coverage and frequency range used. Thus illumination analysis in terms of resolution for the targeted zone becomes important even during the survey planning stage. The wave-equation based illumination analysis and its direct application to two realistic field experiments reveal its important issues and the potential needs in full waveform inversion.

The reciprocal wavefields are identical only if the responses and the orientations of the source and receiver are matched. For the OBS survey, we performed the validation tests based on reciprocity principle and show that the locations of source and receiver should be reciprocal as well as the orientations. Thus, for example, the horizontal mo- tion from the explosive source can be decomposed into two separated horizontal motions generated by vertical and horizontal dipole forces. The reciprocal simulation of horizontal responses can be replicated by summing horizontal and vertical responses from a horizontal dipole source. Such complication occurred mainly related to the type of waveequation used. For second-order wave equation involving only pressure field computations, self-adjoint operator is valid disregarding its orientation. For applications related to first-order pressure-velocity elastodynamic wave equation, special care is needed for waveform simulation, migration and inversion. Concerns about reciprocal wavefields and validation tests are always necessary and even become important for elastic wavefields responses in $2 \mathrm{D}$ and $3 \mathrm{D}$ computations.

Comparing different survey configurations between Figs. 3 and 4, the illumination and resolution analyses indicate that small-offset survey achieves better resolution than large-offset one. On the contrary, the depth of investigation becomes quiet different as wide-aperture survey provides better depth coverage but loses its resolution. For deep structure investigation, large-scale and wide-aperture multi-component seismic survey with less than $1 \mathrm{~km}$ OBS receiver spacing, broad frequency band including low frequency content and combined with MCS data (Wang et al. 2001) becomes inevitably necessary.

Acknowledgements We thank Prof. Jean Virieux for very helpful discussions during his visit to the computational seismology laboratory, Institute of Geophysics, National Central University. We also thank the Associate Editor, T. K. Wang, and C. S. Liu for their efforts and helpful comments in reviewing the manuscript. This project is financially supported under the National Science Council of Taiwan (NSC-096-2917-I-008-109) and the Central Geological Survey (CGS project number: 100-5226904000-02-02). We also appreciate IOC/NTU and IAG/NTOU for their efforts in collecting the MCS and OBS marine dataset respectively. Computations and code developments, tests and implementations were performed with a parallel cluster machine V'Ger in the Center for Computational Geophysics (contribution number NCU-CCG101-0016).

\section{REFERENCES}

Bear, G., C. P. Liu, R. Lu, and D. Willen, 2000: The construction of subsurface illumination and amplitude maps via ray tracing. The Leading Edge, 19, 726-728, doi: 10.1190/1.1438700. [Link]

Berkhout, A. J., L. Ongkiehong, A. W. F. Volker, and G. Blacquière, 2001: Comprehensive assessment of seismic acquisition geometries by focal beams - Part I: Theoretical considerations. Geophysics, 66, 911-917, doi: 10.1190/1.1444981. [Link]

Born M. and E. Wolf, 1980: Principles of Optics, $6^{\text {th }}$ Ed., 
Pergamon, New York.

Červený, V. and I. Pšenčík, 1983: Gaussian beams and paraxial ray approximation in three-dimensional elastic inhomogeneous media. J. Geophys., 53, 1-15.

Červený, V. and L. Klimeš, 1984: Synthetic body wave seismograms for three-dimensional laterally varying media. Geophys. J. R. Astr. Soc., 79, 119-133, doi: 10.11 11/j.1365-246X.1984.tb02845.x. [Link]

Chen, H. W., 1996: Staggered-grid pseudospectral viscoacoustic wave field simulation in two-dimensional media. J. Acoust. Soc. Am., 100, 120-131, doi: 10.11 21/1.415947. [Link]

Chen, H. W., 1998: Three-dimensional geometrical ray theory and modelling of transmitted seismic energy of data from the Nevada Test Site. Geophys. J. Int., 133, 363-378, doi: 10.1046/j.1365-246X.1998.00518. x. [Link]

Chen, H. W., 1999: Three-dimensional acoustic computation of four-component seismic data. Terr. Atmos. Ocean. Sci., 10, 705-718.

Chen, H. W., 2006: Prestack parallel modeling of dispersive and attenuative medium. Terr. Atmos. Ocean. Sci., 17, 203-231.

Chen, H. W. and G. A. McMechan, 1992: Computation of multi-attribute seismic wavefields by solution of the elastodynamic equations. Bull. Seismol. Soc. Am., 82, 1134-1143.

Cheng, W. B., S. S. Lin, T. K. Wang, C. S. Lee, and C. S. Liu, 2010: Velocity structure and gas hydrate saturation estimation on active margin off SW Taiwan inferred from seismic tomography. Mar. Geophys. Res., 31, 77-87, doi: 10.1007/s11001-010-9090-0. [Link]

Claerbout, J. F., 1985: Imaging the Earth's Interior, Blackwell, Pala Alto, CA, 398 pp.

Deng, J. M., T. K. Wang, C. S. Lee, and C. S. Liu, 2010: Origin of gas hydrates imaged from the acoustic basement in the northernmost south China Sea by using OBS. $72^{\text {nd }}$ European Association of Geoscientists \& Engineers Conference \& Exhibition 2010, SP07.

Gelius, L. J., I. Lecomte, and H. Tabti, 2002: Analysis of the resolution function in seismic prestack depth imaging. Geophys. Prospect., 50, 505-515, doi: 10.1046/j.13652478.2002.00331.x. [Link]

Guitton, A., G. Ayeni, and G. Gonzales, 2010: A preconditioning scheme for full waveform inversion. $80^{\text {th }}$ Annual International Meeting, October 17 - 22, 2010, Denver, Colorado, Expanded Abstracts 29, 1008-1012, doi: 10.1190/1.3513017. [Link]

Hoffmann, J., 2001: Illumination, resolution, and image quality of $P P$ - and $P S$-waves for survey planning. The Leading Edge, 20, 1008-1014, doi: 10.1190/1.1487305. [Link]

Husen, S. and E. Kissling, 2001: Local earthquake tomography between rays and waves: Fat ray tomography.
Phys. Earth Planet. Inter., 125, 171-191, doi: 10.1016/ S0031-9201(01)00266-7. [Link]

Iturbe, I., P. Roux, J. Virieux, and B. Nicolas, 2009: Travel-time sensitivity kernels versus diffraction patterns obtained through double beam-forming in shallow water. J. Acoust. Soc. Am., 126, 713-720, doi: 10.1121/ 1.3158922. [Link]

Kang, I. B. and H. W. Chen, 2003: Wavefield simulation in general elastic and viscoelastic fractured media based upon the Pseudo-spectral method. Terr. Atmos. Ocean. Sci., 14, 487-504.

Lecomte, I., H. Gjøystdal, and Å, Drottning, 2003: Simulated prestack local imaging: a robust and efficient interpretation tool to control illumination, resolution, and time-lapse properties of reservoir. $73^{\text {rd }}$ Annual International Meeting, October 26 - 31, 2003, Dallas, Texas, Expanded Abstracts 22, 1525-1528, doi: 10.1190/1.18 17585. [Link]

Liu, C. S., P. Schnürle, Y. Wang, S. H. Chung, S. C. Chen, and T. H. Hsiuan, 2006: Distribution and characters of gas hydrate offshore of southwestern Taiwan. Terr. Atmos. Ocean. Sci., 17, 615-644.

Luo, Y. and G. T. Schuster, 1991: Wave equation inversion of skeletalized geophysical data. Geophys. J. Int., 105, 289-294, doi: 10.1111/j.1365-246X.1991.tb06713.x. [Link]

McMechan, G. A., 1982: Determination of source parameters by wavefield extrapolation. Geophys. J. R. Astr. Soc., 71, 613-628, doi: 10.1111/j.1365-246X.1982.tb 02788.x. [Link]

Muerdter, D. and D. Ratcliff, 2001a: Understanding subsalt illumination through ray-trace modeling, Part 1: Simple 2-D salt models. The Leading Edge, 20, 578-594, doi: 10.1190/1.1438998. [Link]

Muerdter, D. and D. Ratcliff, 2001b: Understanding subsalt illumination through ray-trace modeling, Part 3: Salt ridges and furrows, and the impact of acquisition orientation. The Leading Edge, 20, 803-816, doi: 10.1190/1.1487289. [Link]

Muerdter, D., M. Kelly, and D. Ratcliff, 2001: Understanding subsalt illumination through ray-trace modeling, Part 2: Dipping salt bodies, salt peaks, and nonreciprocity of subsalt amplitude response. The Leading Edge, 20, 688-697, doi: 10.1190/1.1487279. [Link]

Operto, S., J. Virieux, J. X. Dessa, and G. Pascal, 2006: Crustal seismic imaging from multifold ocean bottom seismometer data by frequency domain full waveform tomography: Application to the eastern Nankai trough. J. Geophys. Res., 111, B09306, doi: 10.1029/ 2005JB003835. [Link]

Rickett, J. E., 2003: Illumination-based normalization for wave-equation depth migration. Geophysics, $\mathbf{6 8}, 1371$ 1379, doi: 10.1190/1.1598130. [Link]

Rietveld, W. E. A., A. J. Berkhout, and C. P. A. Wapenaar, 
1992: Optimum seismic illumination of hydrocarbon reservoirs. Geophysics, 57, 1334-1345, doi: 10.1190/ 1.1443200. [Link]

Schneider, W. A. Jr. and G. A. Winbow, 1999: Efficient and accurate modelling of 3-D seismic illumination. $69^{\text {th }}$ Annual International Meeting, October 31 - November 5, 1999, Houston, Texas, SEG Expanded Abstracts, 633-636.

Schuster, G. T. and J. Hu, 2000: Green's function for migration: Continuous recording geometry. Geophysics, $\mathbf{6 5}$, 167-175, doi: 10.1190/1.1444707. [Link]

Virieux, J. and S. Operto, 2009: An overview of full-waveform inversion in exploration geophysics. Geophysics, 74, PWCC1-PWCC26, doi: 10.1190/1.3238367. [Link]

Volker, A. W. F., G. Blacquière, A. J. Berkhout, and L. Ongkiehong, 2001: Comprehensive assessment of seismic acquisition geometries by focal beams - Part II: Practical aspects and examples. Geophysics, 66, 918-931, doi: 10.1190/1.1444982. [Link]

Wang, T. K., K. McIntosh, Y. Nakamura, C. S. Liu, and
H. W. Chen, 2001: Velocity-Interface structure of the southwestern Ryukyu subduction zone from EW95091 OBS/MCS data. Mar. Geophys. Res., 22, 265-287, doi: 10.1023/A:1014671413264. [Link]

Woodward, M. J., 1992: Wave-equation tomography. Geophysics, 57, 15-26, doi: 10.1190/1.1443179. [Link]

Xie, X. B., S. Jin, and R. S. Wu, 2006: Wave-equationbased seismic illumination analysis. Geophysics, 71, S169-S177, doi: 10.1190/1.2227619. [Link]

Zelt, C. A. and R. B. Smith, 1992: Seismic traveltime inversion for 2-D crustal velocity structure. Geophys. J. Int., 108, 16-34, doi: 10.1111/j.1365-246X.1992.tb00836.x. [Link]

Zhu, L., 2000: Crustal structure across the San Andreas Fault, southern California from teleseismic converted waves. Earth Planet. Sci. Lett., 179, 183-190, doi: 10.1016/S0012-821X(00)00101-1. [Link]

Zhu, L., 2002: Deformation in the lower crust and downward extent of the San Andreas Fault as revealed by teleseismic waveforms. Earth Planets Space, 54, 1005-1010. 